Review

\title{
Carcinogenic Effects of Mixtures of Chemicals
}

\author{
Tomoyuki Shirai ${ }^{1}$, Kumiko Ogawa $^{1}$, and Satoru Takahashi ${ }^{1}$ \\ ${ }^{1}$ Department of Experimental Pathology and Tumor Biology, Nagoya City University Graduate School of Medical \\ Sciences, Nagoya 467-8601, Japan
}

\begin{abstract}
Our environment is a sea of chemicals composed of hundreds and thousands of chemicals with great diversity of pharmacological function and structure. Human populations are therefore generally exposed simultaneously to numbers of toxicants, including complex mixtures of chemicals of unknown and variable origin at low doses, some of which are carcinogens and some of which are non-carcinogens. Exposure may occur in different ways and interactions between multiple chemicals acting simultaneously or sequentially in humans are a very important issue for carcinogenic risk assessment and management. Experimental data demonstrate that at very low doses, summation or synergistic enhancement of carcinogenesis is unlikely, particularly in cases of non-genotoxic carcinogens. In this review, we would like to present published data on summation and/or synergistic effects of carcinogens, dependent on the dose applied. (J Toxicol Pathol 2006; 19: 1-13)
\end{abstract}

Key words: summation, synergism, mixture of chemicals, risk evaluation, carcinogenesis

\section{Introduction}

More than 24,000 chemicals are registered for use in Japan. Each year, an estimated 2,000 new ones are introduced as everyday items. Humans are exposed to these chemicals during manufacture, distribution, use and disposal, or when they become pollutants in our air, water, or soil. We do not know the effects of many of these chemicals on our health. Hazard identification, particularly of carcinogenicity, for individual compounds has generally been tested in 2-year standard bioassays using laboratory animals, rats and mice. However, these are tremendously costly as well as time-consuming, so the number of compounds which can be tested is limited. The National Toxicology Program, for example, has been energetically performing chronic carcinogenicity studies on rodents since it start in 1978, but at the present time (as of Nov., 2005), only about 538 chemicals have been examined (TR-1 538) [http://ntp-server.niehs.nih.gov/index.cfm?objectid= 084801F0-F43F-7B74-0BE549908B5E5C1C]. Humans are exposed to thousands of environmental chemicals, and in most instances to multiple chemical compounds simultaneously and/or sequentially. Multiple chemicals may be simple or complex mixtures, as defined by Feron ${ }^{1}$. A simple mixture is a relatively small number of chemicals (ten

Received: 18 January 2006, Accepted: 13 February 2006

Mailing address: Tomoyuki Shirai, Department of Experimental Pathology and Tumor Biology Nagoya City, University Graduate School of Medical Sciences, 1-Kawasumi, Nagoya 467-8601, Japan TEL: 81-52-853-8154 FAX: 81-52-842-0817

E-mail: tshirai@med.nagoya-cu.ac.jp or less), the composition of which is qualitatively and quantitatively known, as with formulated packages of chemicals such as pain killers, gasoline, pesticides, common sources such as tobacco smoke and diesel exhaust. A complex mixture is one that comprises tens, hundreds or thousands of chemicals, the composition of which is qualitatively and quantitatively unknown. Mixtures may also be of widely different chemical complexity, from technical chemical products such as pesticides and dyes, to highly complex mixtures of a series of similar components like polychlorinated biphenyls and coal tars. Since humans are exposed to different compounds including carcinogens throughout life, interactions among chemicals with regard to carcinogenicity are a very important subject for risk evaluation. Summational, synergistic or suppressive responses could be expected; however notwithstanding their importance, there are difficulties in terms of strategy for evaluation of the risks of these interactions. For example, performing a long-term experiment to evaluate carcinogenic hazards of a selected set of multiple compounds may be almost impossible in practice. Alternatives to the long-term carcinogenicity bioassay therefore need to be applied. In this review, we would like to present a limited number of representative data describing alteration of carcinogenic responses in rodents exposed to multiple chemical sets.

\section{Definitions of Terms Related to Carcinogenic Action (in a Certain Organ/Tissue)}

When two or more chemicals act together, carcinogenic responses can be enhanced, suppressed or remain 
unchanged. With enhancement, "summation", "synergism", "syncarcinogenesis" and "co-carcinogenesis", are conceivable. Definitions of these terms were reviewed earlier by Hecker in $1976^{2}$, and Reif further described the definition of synergism with sample data ${ }^{3}$. According to Reif's description, "synergism" is defined as an effect of two or more substances acting together which exceeds the sum of their effects when acting separately. If the end effect of the combined action equals the sum of the effects of the two agents acting independently, the resulting situation is one of "additivity"3. "Syncarcinogenesis" is an augmentational or synergistic action of subcarcinogenic doses of two or more carcinogens; ie., even though exposure to a single chemical does not exert carcinogenicity, if two or more such chemicals act together, an apparent carcinogenic outcome is observed. According to Hecker's review, syncarcinogenesis has two situations depending on the mode of action, pluricarcinogenesis and co-carcinogenesis. Hecker defined pluricarcinogenesis as carcinogenesis by simultaneous or sequential exposure to multiple carcinogens, but this term has not been used recently. Co-carcinogenesis was defined by Hecker as co-action of two or more agents each of which induce events but of different types, one directly and the other only indirectly. In other words, "co-carcinogenic action" is widely accepted when a carcinogen given with or followed by a non-carcinogenic substance results in enhancement of carcinogenicity regardless of the dose of the carcinogen (carcinogenic dose or subcarcinogenic). The term of "summation" is applied to carcinogenic responses which are additive, like " $1+1=2$ "; and the term "synergism" is applied to carcinogenic responses which are more than additive, as in " $1+1>2$ ". In these cases, the timing of the administration of the two or more carcinogens may not be important, and pioneering studies on multiple carcinogens were reported by Hieger ${ }^{4}$ and Lavik et al. ${ }^{5}$

\section{Long-Term Animal Experimentation}

\section{Summation of two chemically unrelated carcinogens}

Nakahara and Fukuoka ${ }^{6}$ investigated the effects of combined treatment with two carcinogens, 4-nitroquinoline $\mathrm{N}$-oxide and 3-methylcholanthrene (originally named as 20methylcholanthrene), each of which has a chemically different nature, on skin carcinogenesis in mice. They found that subcarcinogenic doses of each carcinogen acted additively, resulting in skin cancer development when one was given followed by the other. Their conclusion was that the two carcinogens showed summation of actions and that carcinogenic alteration with either could persist without any recognizable loss for at least 200 days; ie., alteration of cells by each chemical was irreversible.

\section{Synergistic action of two carcinogens, each of which has different organotropism ${ }^{7}$ \\ Montesano et al..$^{7}$ reported that two different carcinogens, diethylnitrosamine, which was given to hamsters sc and found to induce nasal cavity carcinomas,}

and benzopyrene, given intratracheally with ferric oxide, resulted in more carcinomas of the trachea, bronchi and lungs when they were given together.

\section{Synergistic effects of $N$-nitroso compounds (review paper $)^{8}$}

Habs and Schmahl ${ }^{8}$ reviewed the results of long-term animal experiments to investigate additive or synergistic effects of N-nitroso compounds. Carcinogenic nitroso compounds administered to animals together with other carcinogens or non-carcinogens result in the carcinogens working additively or synergistically on tumor induction but in some cases, inhibition occurs. Habs and Schmal discussed the modes of action of pairs of xenobiotics with regard to carcinogenic responses in experimental animals and about the importance of possible organospecific interactions between two compounds for outcomes.

\section{Summation and synergism of multiple carcinogens} all of which have the same organotropism ${ }^{9}$

Four rat urinary bladder carcinogens, N-butyl-n-(4hydroxybutyl)nitrosamine (BBN), N-(4-(5-nitro-2-furyl)-2thiazolyl)formamide (FANFT), N-2-fluorenylacetamide (2AAF) and 3,3'-dichlorobenzidine (3,3'-DCB) were given singly, or in combinations of two or three together to rats at non-carcinogenic doses. The development of transitional cell carcinomas of the urinary bladder was synergistically elevated by treatment with BBN plus FNAFT, 2-AAF and/or $3,3^{\prime}-\mathrm{DCB}$, with the greatest synergistic effect in the case of BBN plus FANFT. However, the combination of three of the carcinogens, BBN, 2-AAF and 3,3'-DCB, did not enhance bladder carcinogenesis over combinations of the pairs, BBN and 2-AAF or BBN and 3,3'-DCB. These results suggest that summation and synergism in carcinogens' action may be dependent on the actual agents, activation and/ or detoxification of carcinogens being mutually affected.

\section{Combinations of very low doses of carcinogens}

Berger et al. ${ }^{10}$ studied syncarcinogenic activity of very low doses of diethylnitrosamine (DEN), Nnitrosopyrrolidine (NPYR) and N-nitrosodiethanolamine (DHPN) in the livers of male rats, performing an entire lifespan experiment with a total of 1,800 rats for this purpose. The rats were sacrified only when moribund. Each carcinogen was given alone at 3 different doses (escalation factor: 3.16), $0.1,0.032$ and $0.01 \mathrm{mg} / \mathrm{kg}$ b.w. for DEN, 0.4 , 0.133 and 0.04 for NPYR and 2.0, 0.63 and 0.20 for DHPN. For combination studies, three different dose levels were set, the high dose combination being composed of the middle doses of individual carcinogens, the middle dose combination being constituted by the lowest doses of each given individually and the low dose combination consisting of one-third of each of the lowest doses. They found that all carcinogens individually as well as in combination induced liver cancers in a dose-dependent manner. Importantly, only the highest dose combination induced an incidence of liver cancers which exceeded the sum of the incidence of each 
Table 1. Liver Tumor Incidences Induced by Combination of the Three Nitrosamines in Rats

\begin{tabular}{|c|c|c|c|c|c|c|c|c|}
\hline \multirow{2}{*}{ Group no. } & \multirow{2}{*}{ No. of animals } & \multirow[t]{2}{*}{ Mode of treatment ${ }^{\mathrm{c}}$} & \multicolumn{3}{|c|}{ Single dose $\mathrm{a}^{\mathrm{a}, \mathrm{b}}$ administered/day } & \multicolumn{2}{|c|}{ Animal with Hepatocellular tumors } & \multirow[t]{2}{*}{$P$ value } \\
\hline & & & DEN & NPYR & DHPN & Carcinomas\% (no.) & Adenomas\% (no.) & \\
\hline 1 & 500 & Control & - & - & - & 0 & $0.2(1)$ & - \\
\hline $2 \mathrm{a}$ & 80 & DEN HD & 0.1 & - & - & $26.3(21)$ & $3.8(3)$ & $<0.001$ \\
\hline $2 b$ & 80 & DEN MD & 0.032 & - & - & $0 \quad(0)$ & $1.3(1)$ & 0.012 \\
\hline $2 \mathrm{c}$ & 80 & DEN LD & 0.01 & - & - & $1.3(1)$ & $1.3(1)$ & 0.005 \\
\hline $3 a$ & 80 & NPYR HD & - & 0.4 & - & $15 \quad(12)$ & $6.3(5)$ & $<0.001$ \\
\hline $3 b$ & 80 & NPYR MD & - & 0.133 & - & $0 \quad(0)$ & $2.5(2)$ & 0.003 \\
\hline $3 c$ & 80 & NPYR LD & - & 0.04 & - & 1.3 (1) & $\begin{array}{ll}0 & (0)\end{array}$ & 0.097 \\
\hline $4 a$ & 80 & DHPN HD & - & - & 2 & $3.8 \quad(3)$ & $1.3(1)$ & $<0.001$ \\
\hline $4 b$ & 80 & DHPN MD & - & - & 0.63 & 1.3 (1) & $\begin{array}{ll}0 & (0)\end{array}$ & $>0.1$ \\
\hline $4 c$ & 80 & DHPN LD & - & - & 0.2 & $0 \quad(0)$ & (0) & $>0.1$ \\
\hline $5 \mathrm{a}$ & 100 & HCD & 0.032 & 0.13 & 0.63 & $13 \quad(13)$ & $3 \quad(3)$ & $<0.001$ \\
\hline $5 \mathrm{~b}$ & 240 & MCD & 0.01 & 0.04 & 0.2 & 2.1 & $1.7(4)$ & $<0.001$ \\
\hline $5 c$ & 240 & LCD & 0.0032 & 0.013 & 0.063 & $0.8 \quad(2)$ & $0.8(2)$ & 0.012 \\
\hline
\end{tabular}

a: $\mathrm{mg} / \mathrm{kg}$ body wt.

(modified from Tables 1 and 2 by Berger et al., 1987)

b: N-nitrosamines were administered via the drinking water $5 \mathrm{x} /$ week.

c: HD, high dose; MD, medium dose; LD, low dose; HCD, high combination dose; MCD, medium combination dose; LCD, low combination dose.

carcinogen (Table 1). The incidence of liver cancers observed with the low dose combination group could not be properly evaluated for summation or synergism of action because there were no data on liver tumor inductions with the doses applied for individual carcinogens. Since liver cancer was found in 1 of 500 untreated rats, it is difficult to properly assess the significance of the incidence in 4 of 240 rats.

\section{Combination effects of 40 carcinogens at low doses in rats}

Takayama et al. carried out a 2-year period animal experiment to explore combined carcinogenic effects of a mixture composed of 40 chemical carcinogens of a variety of types and structures in male rats ${ }^{11}$. The dose of each carcinogen in the diet was $1 / 50$ of the $\mathrm{TD}_{50}$ value and the mixture of chemicals was continuously administered for 102 weeks. The target organs of the individual carcinogens were various, including the liver for $20(50 \%)$ and the Zymbal gland, urinary bladder, thyroid, intestine, skin, hematopoetic organs, spleen, forestomach, kidney and subcutis. Administration of the mixture for 102 weeks resulted in various types of tumor, with significant increases in neoplastic nodules of the liver and thyroid follicular cell adenomas/carcinomas over the non-treated control values. Due to the experimental protocol, it was not clear whether the observed tumorigenic effects on the liver and thyroid were synergistic or additive. Although 20 out of 40 carcinogens had a carcinogenic organ tropism for the liver, the incidence of liver cancer was not elevated in comparison with the incidence rates for any carcinogen given singly. Enhanced thyroid tumor development in the mixture exposed group may have been due to anti-thyroid hormone effects that are thought to be specific tumorigenic actions in rodents ${ }^{12}$. A similar result for three thyroid carcinogens given for 52 weeks at $1 / 3$ of the $\mathrm{TD}_{50}$ value was reported by Hasegawa et al. ${ }^{13}$. A combination of 3 thyroidal carcinogens, 2,4-diaminoanisole sulfate, N, N'diethylthiourea and 4,4'-thiodianiline administered to male F344 rats resulted in development of thyroidal carcinomas in $100 \%$ of the animals, while the incidence rates for the individual chemicals alone were 0 to $10 \%{ }^{13}$. Since the combination significantly suppressed serum levels of thyroxin, and consequently elevated levels of thyroid stimulating hormone from the pituitary, this was suggested to be a major reason for the synergistic enhancement of thyroidal cancer development by the combination treatment ${ }^{13}$.

\section{Short-Term Animal Experimentation}

As mentioned above, execution of long-term experiments to investigate the effects of combinations of multiple chemicals is expensive in terms of both time and money, given the need for many groups of combinations of individual chemicals.

a. The medium-term liver bioassay ${ }^{14}$ : Recently, the medium-term liver bioassay, a 8-week protocol consisting of 2 stages, was established and has become recognized worldwide as an alternative method for the rapid detection of hepatocarcinogenicity of chemicals in the human environment ${ }^{14,15}$. Male F344 rats are given a single intraperitoneal injection of DEN $(200 \mathrm{mg} / \mathrm{kg})$ for initiation of liver carcinogenesis, followed by a 6-week test chemical treatment starting 2 weeks thereafter (Fig. 1). Test chemicals are usually given in the diet or in drinking water and in the 2nd week of test chemical treatment, all rats are subjected to two-third partial hepatectomy for induction of 


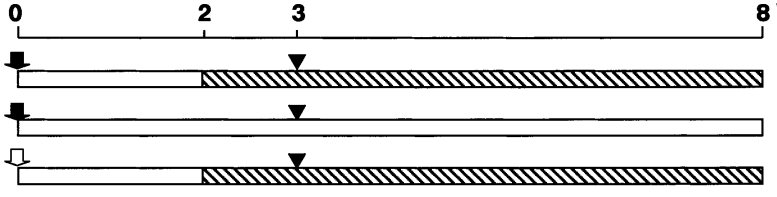

Fig. 1. Experimental protocol for the medium-term liver bioassay. Animals: F344 male rats, 6-week-old
2. DEN (Diethylnitrosamine), $200 \mathrm{mg} / \mathrm{kg}$, i.p.
ㄱ: Saline, i.p.
$\boldsymbol{\nabla}$ : Two-thirds partial hepatectomy
: Test chemical(s) in a diet, drinking water or injection (ig, ip, or sc)
$\square$ : Basal diet

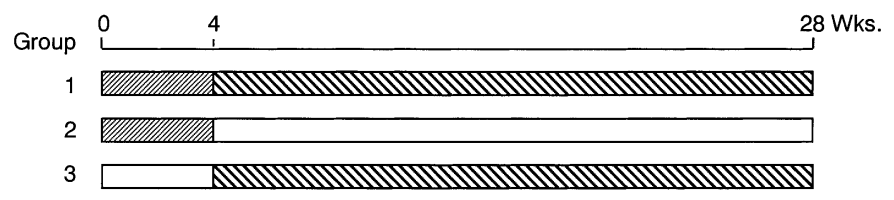

Fig. 2. Experimental protocol for the medium-term multi-organ bioassay

प1דII: DMBDD treatment (DEN, MNU, DMH, BBN, DHPN)

DEN; diethylnitrosamine

MNU; methylnitrosourea

DMH; 1,2-dimethylhydrazine

BBN; butyl-n-(4-hydroxybutyl)nitrosamine

DHPN; N-nitrosodiethanolamine

MIU: Test chemical(s) in a diet, drinking water or injection (ig, ip, or sc) : Basal diet

Table 2. Twenty Organophosphorus Pesticides used in the Medium-Term Liver Bioassay

\begin{tabular}{|c|c|c|c|c|}
\hline Chemical & $\begin{array}{c}\mathrm{ADI} \\
(\mathrm{mg} / \mathrm{kg} / \text { day })\end{array}$ & $\begin{array}{l}\text { Concentration } \\
\text { In the diet (ppm) }\end{array}$ & $\begin{array}{l}\text { Mutagenicity } \\
\text { (Ames test) }\end{array}$ & $\begin{array}{l}\text { Carcinogenicity and } \\
\text { target organ (species) }\end{array}$ \\
\hline \multicolumn{5}{|l|}{ Pesticides (16) } \\
\hline Acephate & 0.03 & 0.3 & + & Liver (mouse) \\
\hline Chlorpyrifos & 0.01 & 0.1 & + & - \\
\hline Chlorfenvinphos & 0.0015 & 0.015 & + & $?$ \\
\hline Dichlorvos & 0.0033 & 0.033 & + & Pancreas (rat), forestomach (mouse) \\
\hline Dimethoate & 0.01 & 0.1 & + & $\begin{array}{l}\text { Digestive tract/system, lung, mammary } \\
\text { gland, ovary (rat and mouse) }\end{array}$ \\
\hline Endosulfan $^{\mathrm{a}}$ & 0.006 & 0.06 & + & - \\
\hline Etrimfos & 0.003 & 0.03 & $?$ & ? \\
\hline Fenitrothion & 0.005 & 0.05 & + & - \\
\hline Isoxathion & 0.003 & 0.03 & - & $?$ \\
\hline Malathion & 0.02 & 0.2 & - & - \\
\hline Methidathion & 0.001 & 0.01 & $?$ & Liver (mouse) \\
\hline Pirimiphos-methyl & 0.01 & 0.1 & ? & $?$ \\
\hline Prothiopphos & 0.0015 & 0.015 & - & $?$ \\
\hline Pyraclofos & 0.001 & 0.01 & $?$ & $?$ \\
\hline Trichlorfon & 0.01 & 0.1 & + & - \\
\hline Vamidothion & 0.008 & 0.08 & + & $?$ \\
\hline \multicolumn{5}{|l|}{ Fungicides (3) } \\
\hline Edifenphos & 0.0025 & 0.025 & - & $?$ \\
\hline Iprobenfos & 0.003 & 0.03 & - & $?$ \\
\hline Tolclofos-methyl & 0.064 & 0.64 & $?$ & $?$ \\
\hline \multicolumn{5}{|l|}{ Herbicides (1) } \\
\hline Butamifos & 0.0016 & 0.016 & $?$ & $?$ \\
\hline
\end{tabular}

(Cited from a Table by Ito et al., 1995)

a: Endosulfan is an organochlorine compound.

?: not reported as far as we know.

regenerative cell replication. The end-point marker is the glutathione S-transferase placental form (GST-P)-positive hepatic focus and the numbers and sizes of GST-P positive foci are semi-quantitatively assessed. This assay system is very sensitive and reliable for the detection of hepatocarcinogenicity and/or promotional effects in a short period $^{14}$.

b. The medium-term multi-organ bioassay: Although the medium-term liver bioassay described above is very sensitive for the detection of hepatocarcinogenicity or liver promoting activity of test compounds, this system is fundamentally confined to the liver. To overcome this weakness medium-term multi-organ bioassays have been established in order to detect carcinogenicity in multiple organs ${ }^{16}$. One of them is the DMBDD model, named by the first letters of the names of 5 carcinogens used in it: DEN, Nmethylnitrosourea (MNU), BBN, 1,2-dimethylhydrazine (DMH) and DHPN. Administration of these 5 carcinogens 


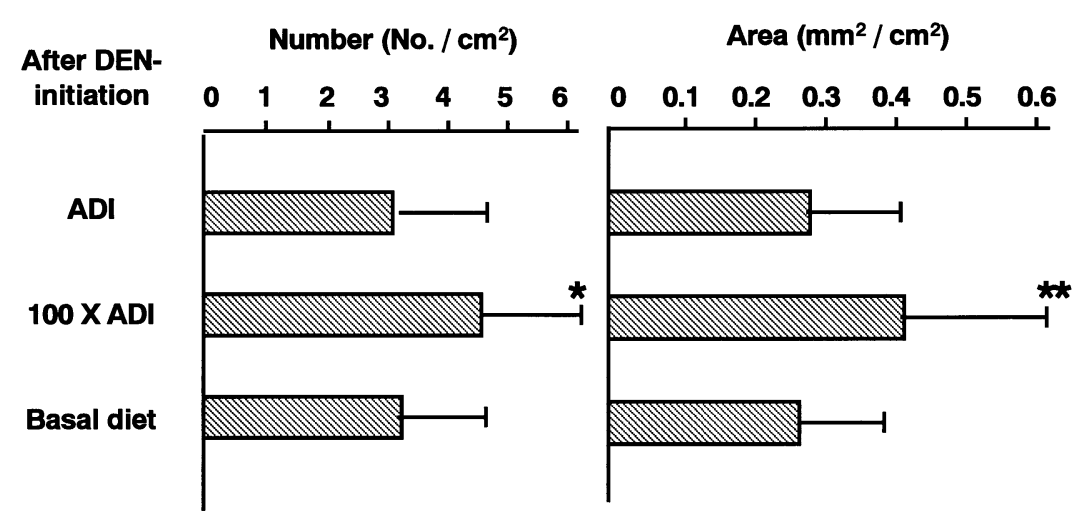

ADI: Acceptable Daily Intake

Fig. 3. Hepatocarcinogenic effects of a mixture of 20 pesticides in the medium-term liver bioassay. The data are numbers and areas of GSTp positive liver cell foci (modified from Ito et al., $1995^{20}$ ). No foci larger than $0.2 \mathrm{~mm}$ in diameter were observed in non-initiated groups. ${ }^{* *},{ }^{*}$ Significantly different from the basal diet group at $\mathrm{P}<0.01, \mathrm{P}<0.05$, respectively.

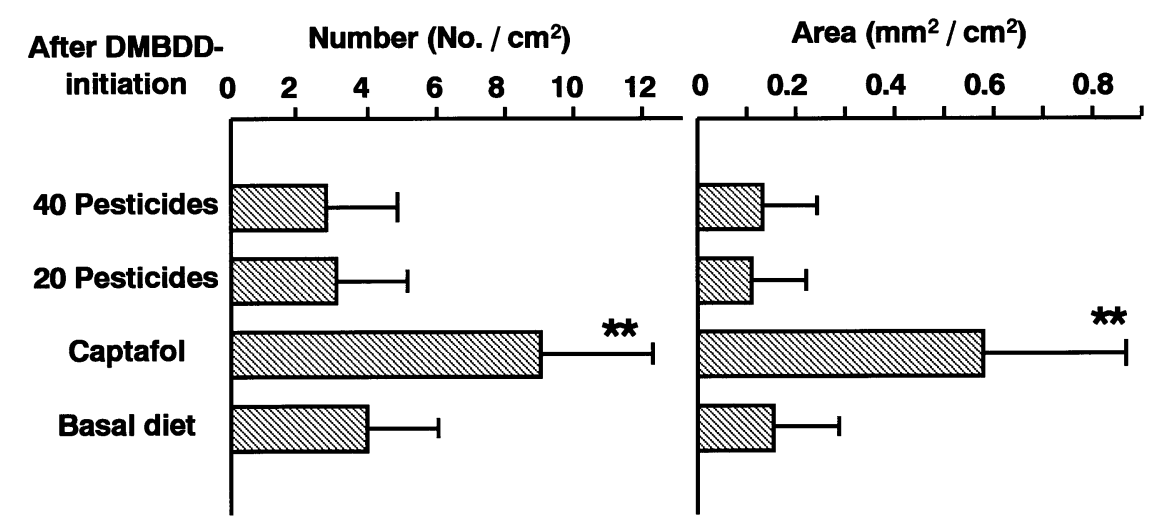

Fig. 4. Hepatocarcinogenic effects of mixtures of 20 or 40 pesticides in the medium-term multi-organ carcinogenicity bioassay. The data are numbers and areas of GST-p positive liver cell foci (modified from Ito et al., 1995 ${ }^{21}$ ). No foci larger than $0.2 \mathrm{~mm}$ in diameter were observed in non-initiated groups. All pesticides were given at $1 / 100$ levels of ADI. **Significantly different from the basal diet group at $\mathrm{P}<0.01$.

for a short period can initiate carcinogenesis in multiple organs, including the liver, urinary bladder, lung, colon and thyroid. F344 rats are given the 5 carcinogens in the order shown in Fig. 2. After this initiation step, rats are exposed to test chemical(s) for 24 or 36 weeks. End-point markers in this system depend on the organ/tissue examined, GST-P positive foci, adenomas and carcinomas for the liver and characteristic preneoplastic and/or neoplastic lesions for the other organs. Using the multi-organ bioassay, promotion or inhibition potentials of possible chemopreventors have been investigated $^{17-19}$.

\section{Effects of a combined administration of 20 pesticides on hepatocarcinogenicity ${ }^{20}$}

In order to clarify the question of whether exposure of agricultural workers and the general population to multiple pesticides might exert summation or synergistic effects at very low doses, the carcinogenic effects of pesticide mixtures were examined with the medium-term liver bioassay.

The twenty pesticides listed in Table 2 were incorporated into diet at individual Acceptable Daily Intake (ADI) levels or 100 times the ADI and given to rats for 6 weeks after DEN initiation. Nineteen of the 20 pesticides examined are organophosphorus compounds. Carcinogenicity including the target organ(s) of each are also provided in Table 2. The safety factor used for ADI by the Japanese Ministry of Health, Labour and Welfare and the Food and Agriculture Organisation of the United Nations (FAO)/WHO is usually 100 (WHO, 1974), and it is usually determined by dividing the no-observed-effect level (NOEL) obtained from laboratory-based chronic toxicity studies by an appropriately chosen safety factor. In other words, the 100 times higher dose levels are the NOELs. As shown in Fig. 3, administration of a mixture of the pesticides at ADI levels did not increase GST-P positive foci in terms of the 
number and area per unit liver section, but individual doses at 100 times the ADI level did significantly enhance the number and area of GST-P positive foci $(\mathrm{P}<0.05$ and 0.01 , respectively). Without DEN initiation, the mixture alone at the level of either ADI or $100 \times$ ADI did not induce GST-P positive foci. These data demonstrate a lack of combination effects at a total dose 100 times higher than the individual ADI levels.

2. Carcinogenic effects of a mixture of 20 and 40 pesticides in the DMBDD medium-term multi-organ carcinogenicity bioassay ${ }^{21}$

In a second experiment, the carcinogenic potential of pesticide mixtures was investigated in the medium-term multi-organ system, the DMBDD model. Two mixtures of pesticides were prepared at individual ADI doses, one comprising 20 and the other 40 as listed in Tables 3 and 4, respectively ${ }^{16}$. There was variation in carcinogenicity and target organs among individual pesticides (Tables 3 and 4). Twenty F344 male rats were given the 5 DMBDD carcinogens within the first 4 weeks and then one of the two mixtures of pesticides was given in the diet for 24 weeks as a test chemical. Control rats were given only the 5 DMBDD carcinogens without pesticides. Neoplastic lesions were histopathologically assessed on paraffin sections along with the appearance of GST-P positive foci in the liver. Interestingly, neither the mixture of 20 nor the mixture of 40 pesticides enhanced the appearance of GST-P positive foci (Fig. 4). The incidences of tumors in the various organs of the groups given either of the mixtures did not significantly differ from those in the controls; sites which had tumor(s) were the thyroid, nasal cavity, lung, oral cavity, forestomach, small intestine, large intestine, liver, kidney, urinary bladder, prostate, seminal vesicles, skin and peripheral nerves ${ }^{16}$.

\section{Effects of mixtures of food-derived heterocyclic amines on hepatocarcinogenesis}

Discovery of mutagenicity for a number of heterocyclic amines (HCAs) which are found in pyrolysates of meat, fish, protein or amino acids caused people to pay more attention to food-derived chemicals as carcinogenic risk factors in our daily life ${ }^{22}$. Subsequently 10 HCAs were proven to possess carcinogenicity in rats and mice and the possible contribution of this group of compounds to human cancer development has become a big issue because of the risk of repeated exposure in daily life through food intake.

In order to clarify potential synergism among multiple HCAs at low doses with regard to hepatocarcinogenesis, combined administration of carcinogenic HCAs was performed in three animal experiments using the mediumterm liver bioassay system: 2 experiments with $5 \mathrm{HCAs}^{23,24}$ and one with $10 \mathrm{HCAs}^{25}$.

In the first experiment ${ }^{23,25}$, potential synergism between five HCAs at low doses was evaluated. 3-Amino-1,4dimethyl-5H-pyrido[4,3-b]indole (Trp-P-1, 150 ppm.), 2aminodipyrido[1,2-a:3',2'-d]imidazole (Glu-P-2, 500 ppm), 2-amino-3-methylimidazo[4,5-f]quinoline (IQ, 300 ppm), 2amino-3-methylimidazo[4,5-f]quinoline (MeIQ, 300 ppm.), and 2-amino-3,8-dimethylimidazo[4,5-f]quinoxaline (MeIQx, 400 ppm.) were incorporated into diet, individually at the carcinogenic dose, or at $1 / 5$ or $1 / 25$ of this. Other groups received the five chemicals in combination, each at the $1 / 5$ or $1 / 25$ levels. The numbers of GST-P positive foci were significantly increased with all chemicals at the highest dose, as expected, and Trp-P-1, IQ and MeIQ also exerted positive influences even at the $1 / 5$ dose level (Fig. 5a). Similar results were obtained regarding areas of the foci at the highest dose levels, with the exception of Glu-P-2. An increase was also observed for MeIQ at the 1/5 dose. Additive or synergistic effects between the HCAs were evident in the groups given both the $1 / 5$ and $1 / 25$ dose levels, development of GST-P positive foci being increased over the sum totals of individual data for the $1 / 5$ or $1 / 25$ dose groups (Fig. 5a and 5b). All five HCAs tested increased development of GST-P positive foci in a dose-dependent manner and synergistic carcinogenic effects were apparent at the low dose level ( $1 / 5$ dose level) but not at the very low doses (1/25 dose level).

In the second experiment ${ }^{24,25}$, a different combination of 5 HCAs was employed: 3-amino-1-methyl-5Hpyrido[4,3-b]indole (Trp-P-2, $500 \mathrm{ppm}), 2$-amino-6methyldipyrido[ $\left.1,2-a: 3^{\prime}, 2^{\prime}-d\right]$-imidazole (Glu-P-1, 500 ppm), 2-amino-3-methyl-9H-pyrido[2,3-b]indole (MeA $\alpha \mathrm{C}$, 800 ppm), 2-amino-9H-pyrido[2,3-b]indole (A $\alpha \mathrm{C}, 800$ ppm), and 2-amino-1-methyl-6-phenylimidazo[4,5$b$ ]pyridine (PhIP, $400 \mathrm{ppm}$ ). As in Experiment 1, each HCA was given at the dose used in earlier carcinogenicity assays (above doses), and at $1 / 5$ or $1 / 25$ of these, or all 5 chemicals together, each at the $1 / 5$ or $1 / 25$ levels. The numbers and areas of GST-P-positive foci were significantly elevated by all the HCAs, except for PhIP, at the highest dose, the results being consistent with their reported liver carcinogenicities. In the combined treatment at the $1 / 5$ dose levels, synergistic enhancement occurred; the numbers and areas of foci were significantly increased above the sums of the individual data (Fig. 6a and 6b). However, this was not the case for the 1/25 dose groups.

The third experiment featured a combination of 10 HCAs (2 combinations composed of 5 different HCAs) ${ }^{25}$. Five carcinogenic HCAs, Trp-P-1(150 ppm.), Glu-P-2 (500 ppm), IQ (300 ppm) and MeIQ (300 ppm) for the first subgroup, and MeIQx (400 ppm), and Trp-P-2 (500 ppm), Glu-P-1(500 ppm), MeA $\alpha \mathrm{C}$ (800 ppm), A $\alpha \mathrm{C}$ (800 ppm) and $\mathrm{PhIP}$ (400 ppm) for the second subgroup were administered together or individually in diet at levels of $1 / 1,1 / 5$ or $1 / 25$ of carcinogenic doses (Fig. 7), and all 10 chemicals were given at $1 / 10$ or $1 / 100$ levels. Induction of GST-P positive foci was increased in some combination groups over the sums of the effects for the individual groups at the same doses (apparent synergism). This was most obvious in the group given all 10 chemicals at the $1 / 10$ dose levels. However, it is of interest that the values of the GST-P positive foci in the combined groups were generally very close to the averages 
Table 3. Information Summary for 20 Pesticides for which Carcinogenicity has been Reported or Suspected

\begin{tabular}{llcl}
\hline Pesticides & $\begin{array}{c}\text { ADI } \\
\text { (mg/kg/day) }\end{array}$ & $\begin{array}{c}\text { Mutagenicity } \\
\text { (Ames test) }\end{array}$ & \multicolumn{1}{c}{$\begin{array}{c}\text { Carcinogenicity and } \\
\text { target organs (species) }\end{array}$} \\
\hline $\begin{array}{l}\text { Insecticides (9) } \\
\text { Acephate }\end{array}$ & 0.03 & + & Liver (mouse) \\
Dichlorvos & 0.0033 & + & $\begin{array}{l}\text { Pancreas, leukemia (rat), stomach (mouse) } \\
\text { Dicofol }\end{array}$ \\
Cypermethrin & 0.025 & - & Liver (mouse) \\
Permethrin & 0.05 & $?$ & Lung (mouse) \\
Phosmet & 0.048 & $?$ & Liver, lung (mouse) \\
Amitraz & 0.02 & + & Liver (mouse) \\
Clofentezine & 0.012 & $?$ & Suspected \\
Propoxur & 0.086 & $?$ & Suspected \\
Herbicides (5) & 0.063 & $?$ & Suspected \\
2,4-D & & & \\
Glyphosate & 0.3 & $-/+$ & Brain (rat) \\
Trifluralin & 0.15 & - & Kidney (mouse) \\
Mefolachlor & 0.0075 & - & Multiple organs (rat, mouse) \\
Dichlobenil & 0.097 & $?$ & Positive (rat) \\
Fungicides (6) & 0.004 & $?$ & Suspected \\
Captafol & & & \\
Propiconazole & 0.05 & $-/+$ & Multiple organs (rat, mouse) \\
Fosetyl & 0.018 & $?$ & Liver (mouse) \\
Triadimefon & 0.88 & $?$ & Urinary bladder (rat) \\
Mancozeb & 0.012 & $?$ & Suspected \\
Maneb & 0.05 & $?$ & Suspected \\
\hline & 0.005 & $?$ & Suspected \\
\hline
\end{tabular}

?: not reported as far as we know.

Table 4. Information Summary for 40 Pesticides in High Volume Production

\begin{tabular}{|c|c|c|c|c|c|c|c|}
\hline Pesticides & ADI (mg/kg/day) & Ames test & Carcinogenicity $^{\mathrm{a}}$ & Pesticides & ADI (mg/kg/day) & Ames test & Carcinogenicity $^{\mathrm{a}}$ \\
\hline \multicolumn{4}{|l|}{ Insecticides (17) } & \multicolumn{4}{|l|}{ Herbicides (10) } \\
\hline Acephate & 0.03 & + & Liver (mouse) & Glyphosate & 0.15 & - & Kidney (mouse) \\
\hline Chlorobenzilate & 0.02 & $?$ & Liver (mouse) & Metolachlor & 0.197 & $?$ & Positive (rat) \\
\hline Cypermethrin & 0.05 & $?$ & Lung (mouse) & Sethoxydim & 0.14 & $?$ & - \\
\hline Permethrin & 0.048 & $?$ & Liver, lung (mouse) & Thiobencarb & 0.009 & $?$ & - \\
\hline Clofentezine & 0.0086 & $?$ & Suspected & Peendimethalin & 0.043 & $?$ & - \\
\hline Oxamyl & 0.02 & $?$ & - & Metribuzin & 0.0125 & $?$ & - \\
\hline Chlorpyrifos & 0.01 & $?$ & - & Quinclorac & 0.029 & $?$ & $?$ \\
\hline Cyhalothrin & 0.0085 & $?$ & - & Chlorpropham & 0.1 & $?$ & $?$ \\
\hline Diflubenxuron & 0.012 & $?$ & - & Bensulide & 0.04 & $?$ & $?$ \\
\hline Pirimiphos-methyl & 0.01 & $?$ & - & Bentazone & 0.09 & $?$ & $?$ \\
\hline Bendicarb & 0.004 & $?$ & - & Fungicides (12) & & & \\
\hline Malathion & 0.02 & $?$ & - & Propiconazole & 0.018 & $?$ & Liver (mouse) \\
\hline Fenbutantin oxide & 0.03 & $?$ & $?$ & Manab & 0.005 & $?$ & Suspected \\
\hline Cyfluthrin & 0.02 & $?$ & $?$ & Triadimefon & 0.012 & $?$ & Suspected \\
\hline Trichlorfon & 0.01 & $?$ & $?$ & Imazalil & 0.025 & $?$ & - \\
\hline Fenzalerate & 0.02 & $?$ & $?$ & Zineb & 0.005 & $?$ & - \\
\hline Flucythrinate & 0.0125 & $?$ & $?$ & Vinclozolin & 0.1215 & $?$ & - \\
\hline \multicolumn{4}{|c|}{ Plant growth regulator (1) } & Fenarimol & 0.01 & $?$ & - \\
\hline \multirow[t]{5}{*}{ Mepiquat chloride } & 0.075 & $?$ & $?$ & Flutolanil & 0.08 & $?$ & - \\
\hline & & & & Metalaxyl & 0.019 & $?$ & - \\
\hline & & & & Chinomethionat & 0.006 & $?$ & $?$ \\
\hline & & & & Pyrifenox & 0.1 & $?$ & $?$ \\
\hline & & & & myclobutanil & 0.012 & $?$ & $?$ \\
\hline
\end{tabular}

a: Target organs (species).

(Cited from a Table in the paper by Ito et al., 1995)

?: not reported as far as we know. 

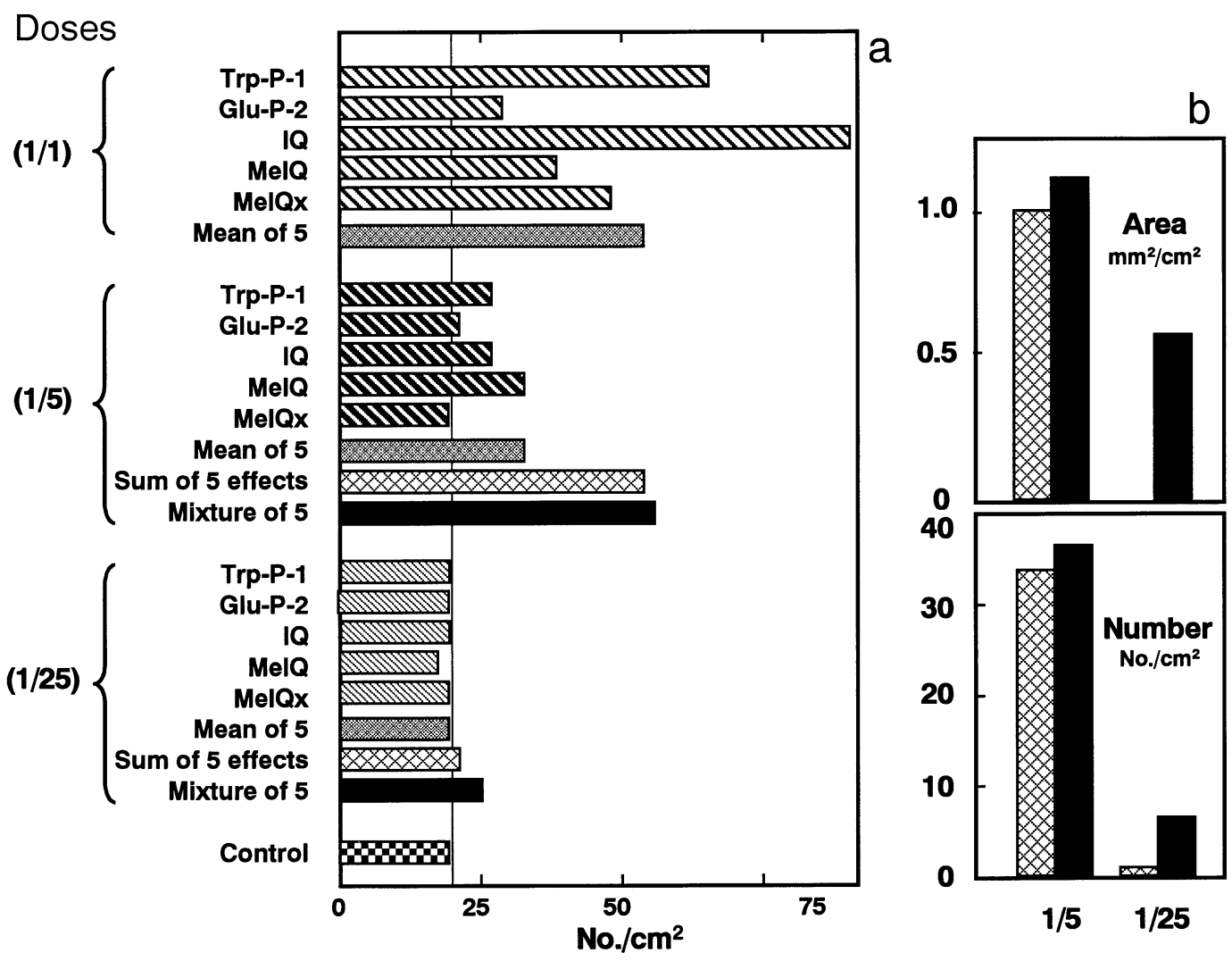

Fig. 5. Synergistic carcinogenic effects of five heterocyclic amines (HCAs), Trp-P-1, Glu-P-2, IQ, MeIQ and MeIQx, given together at $1 / 5$ and $1 /$ 25 of full dose levels, on the induction of GST-P positive foci. The HCAs were also individually given at the full dose, and 1/5 and 1/25 full dose levels(a). Quantitative analysis, performed using net values obtained by subtracting the background levels (control values in group 2) from the measured numbers and areas from each subgroup, shows apparent synergistic effects on both numbers and areas of GST-P positive foci by the 'mixture of 5', especially at the 1/25 carcinogenic levels(b) ${ }^{25}$. Modified from Ito et al., $1991^{23,25}$.

Table 5. Numbers and Areas of GST-P Positive Foci in the Livers of Rats without Prior DEN Initiation ${ }^{\mathrm{a}}$

\begin{tabular}{lcccc}
\hline Treatment & $(\text { dose })^{\mathrm{b}}$ & No. of rats & Number $\left(\mathrm{No} . / \mathrm{cm}^{2}\right)^{\mathrm{c}}$ & Area $\left(\mathrm{mm}^{2} / \mathrm{cm}^{2}\right)$ \\
\hline Trp-P-1 & $(1 / 1)$ & 10 & $1.38 \pm 0.63$ & $1.38 \pm 0.63$ \\
Glu-P-2 & $(1 / 1)$ & 10 & $0.13 \pm 0.21$ & $0.13 \pm 0.21$ \\
1Q & $(1 / 1)$ & 10 & $4.82 \pm 1.62$ & $4.82 \pm 1.62$ \\
MelQ & $(1 / 1)$ & 10 & $0.38 \pm 0.74$ & $0.38 \pm 0.74$ \\
MelQx & $(1 / 1)$ & 9 & $2.20 \pm 1.10$ & $2.20 \pm 1.10$ \\
Trp-P-1 & $(1 / 5)$ & 10 & $0.19 \pm 0.26$ & $0.19 \pm 0.26$ \\
Glu-P-2 & $(1 / 5)$ & 10 & $0.08 \pm 0.12$ & $0.08 \pm 0.12$ \\
1Q & $(1 / 5)$ & 9 & $0.50 \pm 0.27$ & $0.50 \pm 0.27$ \\
MelQ & $(1 / 5)$ & 10 & $0.39 \pm 0.38$ & $0.39 \pm 0.38$ \\
MelQx & $(1 / 5)$ & 10 & $0.04 \pm 0.12$ & $0.04 \pm 0.12$ \\
Combined & $(1 / 5)$ & 12 & $1.44 \pm 0.65$ & $1.44 \pm 0.65$ \\
Trp-P-1 & $(1 / 25)$ & 10 & 0 & 0 \\
Glu-P-2 & $(1 / 25)$ & 10 & $0.44 \pm 0.11$ & $0.44 \pm 0.11$ \\
1Q & $(1 / 25)$ & 10 & $0.07 \pm 0.15$ & $0.07 \pm 0.15$ \\
MelQ & $(1 / 25)$ & 10 & $0.03 \pm 0.10$ & $0.03 \pm 0.10$ \\
MelQx & $(1 / 25)$ & 9 & $0.19 \pm 0.29$ & $0.19 \pm 0.29$ \\
Combined & $(1 / 25)$ & 12 & $0.16 \pm 0.21$ & $0.16 \pm 0.21$ \\
\hline
\end{tabular}

See text for abbreviations of each chemical.

a: Foci $>0.1 \mathrm{~mm}$ in diameter were quantified.

b: Relative to the dose levels used in carcinogenicity

c: Mean \pm SD 

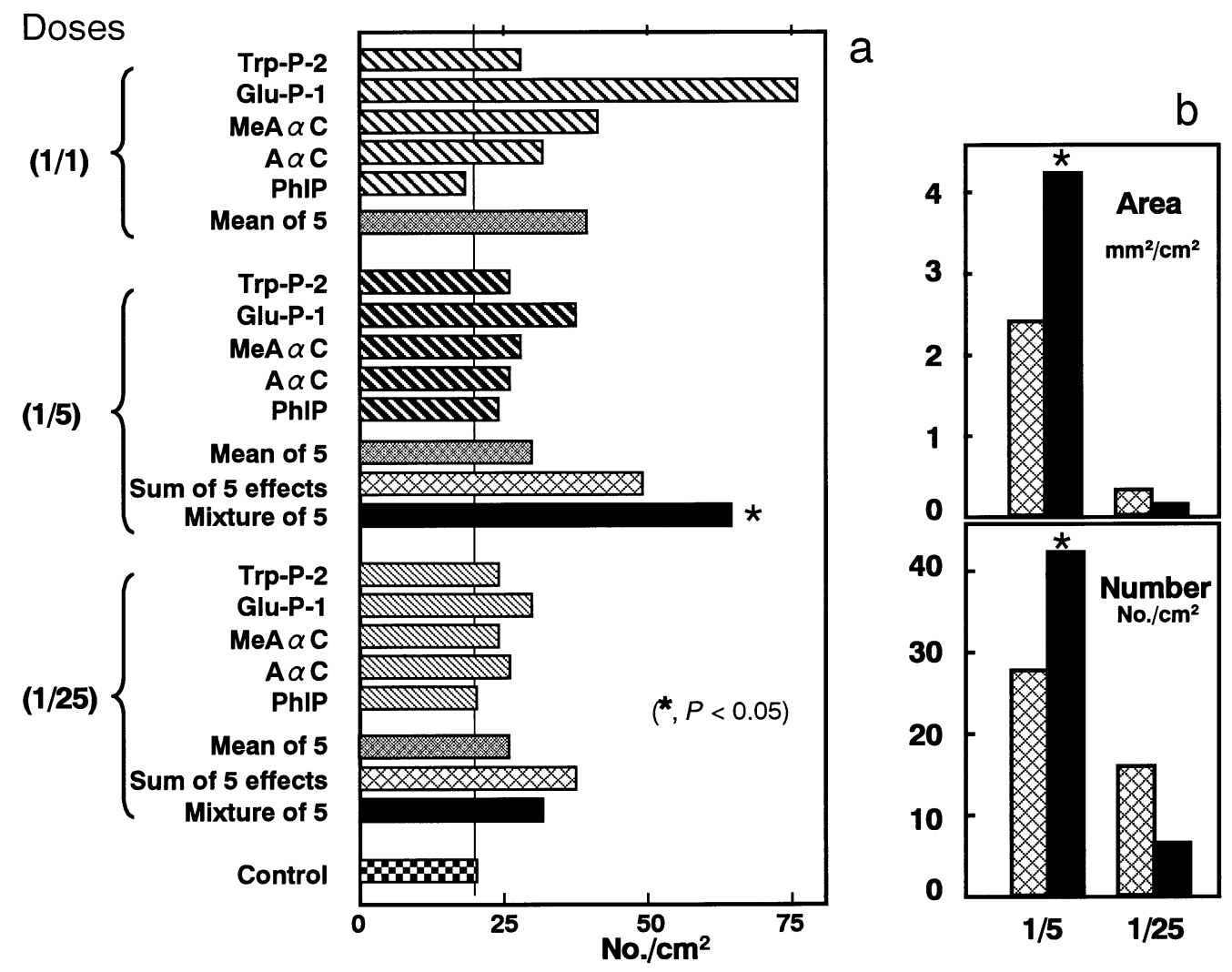

Fig. 6. Combined effects of 5 heterocyclic amines (HCAs) Trp-P-2, Glu-P-1, MeA $\alpha \mathrm{C}, \mathrm{A} \alpha \mathrm{C}$ and PhIP, given together at 1/5 and 1/25 full dose levels, on the induction of GST-P positive foci. The HCAs were also individually given at full dose, and 1/5 and 1/25 full dose levels. Results for the 'mixture of 5' at 1/5 levels were close to the 'mean of 5' were at the full doses. Similarly, the results for the 'mixture of 5' at $1 / 25$ levels are close to the 'mean of 5 ' at $1 / 5$ levels(a) $)^{25}$, although the values for the $1 / 25$ levels singly are almost the same as the control value, indicating apparent synergism. Modified from Hasegawa et al., 199124.

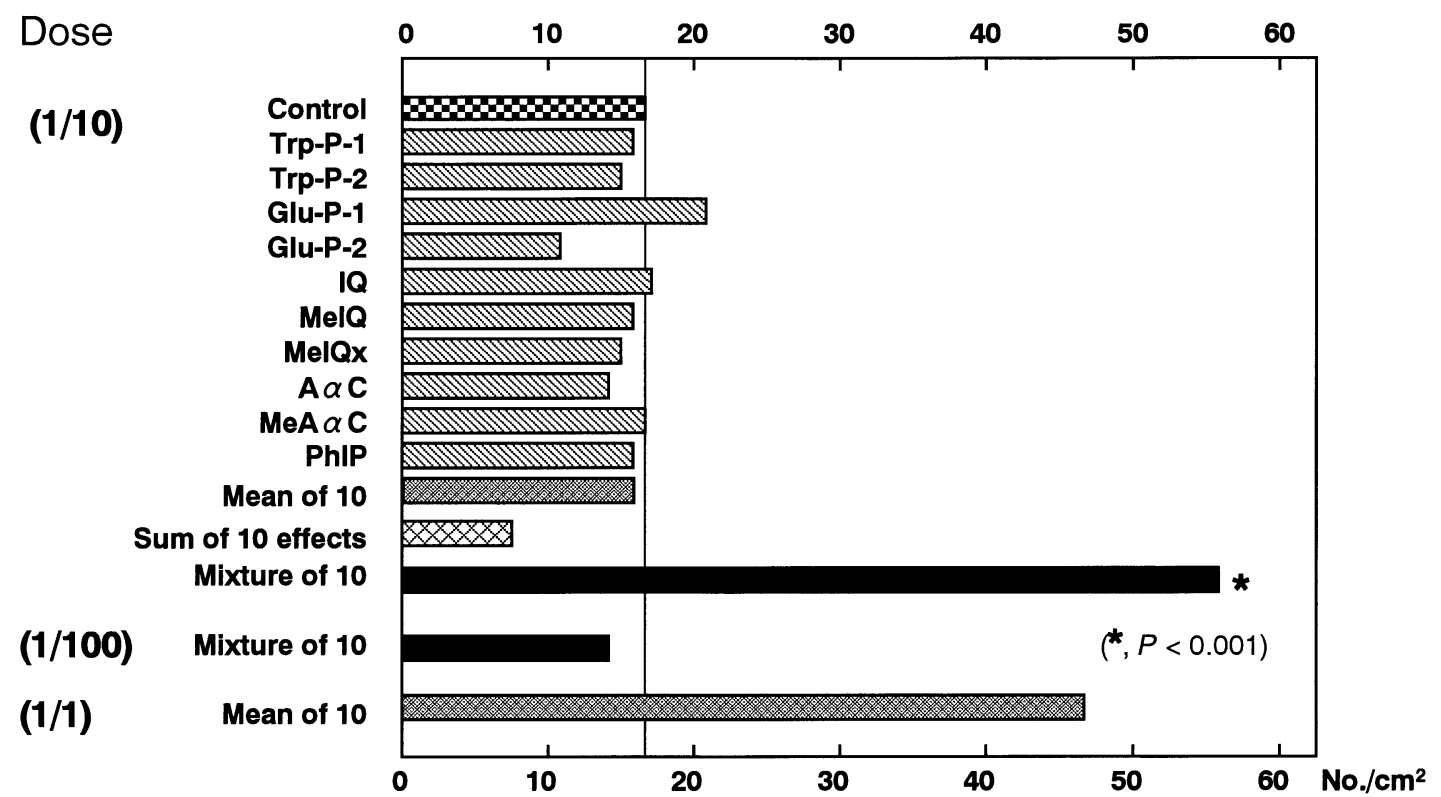

Fig. 7. Combined effects of 10 heterocyclic amines on numbers of GST-P-positive hepatic foci. Effects of individual chemicals at the $1 / 10$ levels were essentially absent. When mixed in the same diet, enhancement was evident at the $1 / 10$ dose level when evaluated by the additive model. However, the result for the 'mixture of 10 ' at the $1 / 10$ level was only slightly higher than the mean of the values for the 10 compounds administered individually at the full dose. The full dose data are cited from the first two experiments. See text for abbreviations for each chemical. Cited from Hasegawa et al., 199124,25. 
of five or 10 individual results for the corresponding higher dose groups, indicating iso-additivity of HCA effects (Fig. 7). True (strict) synergism, however, was expected for the results of groups including PhlP and Trp-P-2 in combination, since they are non-hepatocarcinogenic but induce a key metabolic enzyme for HCAs (CYP1A2). The details of the results can be referred to in the original paper $^{25}$.

\section{Organ-specificity of combined effects of carcinogens and modifiers of carcinogensis in a three carcinogen medium-term multi-organ model ${ }^{26}$}

Combined effects of low doses of not only carcinogens but also modifiers of carcinogenesis on tumor development were assessed using a complex medium-term multi-organ model in F344 rats. In this assessment, three initiating carcinogens (DEN, MNU and DHPN) were used instead of the 5 carcinogens of the DMBDD model. Three combinations as test chemicals were prepared as follows: (1) a group of known hepatocarcinogens (2-AAF, Ndimethylnitrosamine (DMN), 3'-Me-DAB, phenobarbital and thioacetamide); (2) a group of nitroso compounds (BBN, $\mathrm{N}$-dibutylnitrosamine $\quad(\mathrm{DBN}), \quad \mathrm{N}$-ethyl-Nhydroxyethylnitrosamine (EHEN), N-methyl-N'-nitro-Nnitrosoguanidine (MNNG) and N-propylnitrosourea (PNU)) having various target organ specificities; and (3) a group of antioxidants (butylated hydroxyanisole (BHA), catechol, propyl gallate and tert-butylhydroquinone (TBHQ)) having various inhibiting or enhancing activities depending on the target organ. Doses used were generally below the known effective level for the individual chemicals. Although the hepatocarcinogen group in combination with various nitroso compounds increased the incidences of liver tumors (hepatocellular adenomas and carcinomas), liver tumor incidences were clearly reduced when the group of hepatocarcinogens and/or the group of nitroso compounds were administered in combination with the groups of antioxidants. For the urinary bladder, however, the combination with the nitroso compounds and the antioxidants enhanced cancer development, and the addition of the hepatocarcinogens further increased tumorigenesis. An additive effect on the numbers of preneoplastic lesions in rat gastric mucosa was evident in the combination of the antioxidants and the nitroso compounds. No synergistic effects on tumor development were seen in other organs. These results demonstrate that combinations of various compounds at low doses can additively or synergistically exert either enhancing or inhibitory effects on the development of preneoplastic and neoplastic lesions in different organs in a single model.

\section{Discussion}

Human populations are generally exposed simultaneously to a number of toxicants present in the environment, including complex mixtures of chemicals, carcinogens and non-carcinogens, of unknown and variable origin at low doses. Practical exposure is to numerous kinds of chemicals differing in terms of structure, chemical nature and dosage, as with tobacco smoke.

The strength of carcinogenicity of a chemical varies with the dosage, and dose-dependent increase of tumor development due to a single carcinogenic substance can follow linear, J-shaped or exponential curves. It can be considered that these carcinogenic responses result from summation or synergistic responses to two, three or four units of a unit dose of a chemical. Thus, if two or more chemicals that have the same organ tropism and same carcinogenic mechanisms act together, it is easy to understand that summation or synergistic response occurs as with dose-related responses for a single chemical.

While scientific methods for evaluating the potential carcinogenic risks of pure compounds are relatively well established, methods for assessing the risks of complex mixtures are somewhat less developed. Mixtures, such as diesel emissions and tobacco smoke, have been epidemiologically shown to have carcinogenic potential, but because of the difficulty of establishing appropriate methods to represent the human exposure situation, ie., inhalation through the respiratory tract, no experimental proof of lung carcinogenicity of tobacco smoke in animals has been obtained although carcinogenicity of individual components such as benz[a]pyrene, cadmium, 2-naphthylamine and nitrosamines have been individually confirmed by administration intratracheally, orally or subcutaneously. Hutt et al. reported in 2005 the first study to demonstrate a robust increase in lung cancer in mice after a lifetime of inhalation exposure to a mainstream of cigarette smoke $\mathrm{e}^{27}$. Their report clearly demonstrated that an airborne complex mixture to which human beings are exposed through the respiratory tract can be properly studied in experimental animals even though there are some limitations ${ }^{28}$. For appropriate risk evaluation by inhalation of airborne complex mixtures as a whole, the system needs to be highly sophisticated in order to control exposure levels not only to experimental animals but also workers and/or scientists and the surrounding environment. Problems with budget, space and safety would limit the number of institutions in which such a system could be set up. Therefore, there are very practical limitations for performing inhalation studies to evaluate airborne mixtures.

Conventional long-term rodent bioassays are usually applied to the evaluation of certain mixtures of compounds, but again they require time and money and a good facility. Because human exposure levels to most environmental toxicants are very low, there is a need to evaluate the potential carcinogenic risks of multiple exposures at low levels of exposure. Long-term experimentation, the best being a lifespan exposure, is therefore required for precise evaluation of summation or synergistic effects of multiple compounds at low levels. This kind of animal experiment is exceptionally large scale with a long time period, running into constraints of space and research funding.

As alternative protocols for assessment of carcinogenic effects of mixtures, animal models featuring initiation and 
promotion with a relatively short-term experimental period are applicable as time and cost saving methods. As well demonstrated by researchers in Ito's group ${ }^{20,21,23-26}$, the medium-term liver bioassay and the medium-term multiorgan model are plausible alternatives to long-term studies. Particularly, in the medium-term liver bioassay, the endpoints, the numbers and areas of GST-P positive liver foci, allow very sensitive evaluation of the tumorigenicity of test compounds in the liver, and this model is particularly appropriate for investigating summation, synergism, or inhibition of carcinogenic responses with mixtures of multiple chemicals.

As reviewed here, experiments carried out exploring interactions of two or more carcinogenic chemicals given simultaneously under various conditions have featured combinations of carcinogens with differences in organotropism $^{7,25}$, the same organotropism ${ }^{9,13,24}$, and similar $8,13,24,25$ or dissimilar ${ }^{6,26}$ chemical natures. As noted above, mixtures of chemicals for testing can be obtained in a number of ways, and the selection will depend primarily on the aim of the study to be conducted. Samples obtained from the environment are more relevant to the circumstances of human exposure and those samples are suitable for assessing the potential effects of a specific mixture found in the environment of an exposed population. However, samples derived directly from the environment are very complicated, and furthermore they must be processed to a form usable for testing in laboratory by concentration, dilution, filtration, or sterilization. In addition, mixtures in the field change over time and one field sample may not represent average exposures. In addition, identification of the active components in mixtures is important for purposes of risk management. If the mixture is derived from a complicated manufacturing process or certain food components, it may be very difficult to synthesize a representative substitute mixture in the laboratory. On the other hand, laboratoryderived mixtures can be controlled in their composition, thus allowing replication of experiments.

As reviewed here, summation and/or synergistic effects with mixtures of carcinogens have often been found evident at high exposure levels. However, at low dose levels, this is not always the case; thus significant findings were that a low dose combination of three carcinogenic nitrosamines did not exert summation or synergism regarding tumor development ${ }^{10}$, and a combination of 20 or 40 pesticides at ADI levels resulted in no enhancement of hepatocarcinogenesis, but at doses at the NOEL, removing the safety factor of $\times 100$, synergistic enhancement of GST-P positive foci was demonstrated ${ }^{21}$. From the theoretical point of view, the safety factor reflects both the possibility of an increased sensitivity of humans relative to laboratory animals and the variation in susceptibility within the human population, and that for ADI used by the Japanese Ministry of Health, Labour and Welfare and the FAO/WHO is usually 100. The data clearly show that exposure to multiple nongenotoxic chemicals at ADI levels entails practically very low risk in humans. Another significant finding is that the combination of 5 or 10 carcinogenic HCAs did not exert summation or synergistic tumor induction when given at $1 /$ 25 or $1 / 100$ carcinogenic doses ${ }^{23-25}$. These findings suggest that at very low doses, exposure to multiple chemicals may not be risk factors for human populations at least under the conditions conducted in animal experiments. Genotoxic carcinogens like nitrosamines and HCAs induce DNA damage through formation of DNA adduct(s) and/or oxygen radical species produced as bi-products and alteration of the genome is thought to be irreversible once it has take place, leading to the assumption that risks may be additive even at low doses. The data demonstrated by Burger ${ }^{10}$ and Ito's group $^{23-25}$, however, provide evidence that combination treatment with genotoxic carcinogens at low doses does not necessarily entail additive risk for carcinogenicity. This phenomenon could be partly explained by detoxification of carcinogens administered and/or successful repair of DNA damage caused by low doses of carcinogens. Thus, it is implied that additivity should not be considered a universal phenomenon that occurs without exception. Presumably the discrepancy found in the combined effects between the 2 sets of five HCAs is a good example of the complexity of interactions among multiple carcinogens.

Fukushima et al. ${ }^{29}$ recently proposed the presence of a practical threshold for carcinogenicity of non-genotoxic carcinogens. Their many large scale animal experiments on dose related responses have used not only precancerous lesions as end-points but also other parameters such as oxidative stress, DNA repair, cell proliferation, apoptosis, intracellular communication and cell signaling. According to their data, at very low dose(s) some non-genotoxic carcinogens even exert inhibitory effects on carcinogenesis rather than promotion, a phenomenon refered to as hormesis. Their findings for the presence of a threshold and hormesis are in line with the data showing neither summation nor synergism in hepatocarcinogenesis induced by 20 or 40 pesticides given together at the ADI dose levels (Fig. 5 and Tables 4 and 5).

\section{Conclusions}

Experimental data demonstrate that at very low doses, summation or synergistic enhancement of carcinogenesis is unlikely, even in cases of genotoxic carcinogens. However, because of the possible great diversity of pharmacological functions and structures, interactions of multiple chemicals are not simple to elucidate. Carcinogenic activities of chemicals are, for example, conceptually composed of actions in two processes, initiation and promotion. Chemical substances active in both of these are referred to as complete carcinogens, but the ratio between the initiation and promotion or strength of each activity can vary from chemical to chemical. Chemicals with only initiation activity are referred to as initiators and ones with only promotion activity as promoters ${ }^{30}$. Furthermore, there are many chemicals that possess the potential to modify the activities of the enzymes responsible for activating or 
detoxifying xenobiotics which in turn, would modify the carcinogenic potentials of chemicals taken into the body. It is well-known that food nutrients and/or components are also capable of altering the activities of such enzymes. Chemical carcinogenesis is also dependent on numerous processes including absorption, distribution, activation, and detoxification of the chemical besides targeted cellular alterations, making for very great complexity when mixtures of compounds are involved. In addition to practical bioassays for carcinogenicity of certain simple or complex mixtures, the elucidation of mechanisms underlying the outcomes based on the modes of action of component chemicals should be carried out to obtain a better understanding of mixture-carcinogenesis.

Acknowledgement: This work was supported in part by the Ministry of Health, Labour and Welfare of Japan and the Society for the Promotion of Pathology of Nagoya, Japan.

\section{References}

1. Feron VJ, Groten JP, and van Bladeren PJ. Exposure of humans to complex chemical mixtures: hazard identification and risk assessment. Arch Toxicol Suppl. 20: 363-373. 1998.

2. Hecker E. Definitions and terminology in cancer (tumor) etiology - an analysis aiming at proposals for a current internationally standardized terminology. Gann. 67: 471481. 1976.

3. Reif AE. Synergism in carcinogenesis. J Natl Cancer Inst. 73: 25-39. 1984.

4. Hieger I. On the mechanism of carcinogenesis by chemical compounds. Am J Cancer. 28: 522-525. 1936.

5. Lavik PS, Moore PR, Rusch HP, and Baumann CA. Some additive effects of carcinogenci hydrocarbons. Cancer Res. 2: 189-192. 1942.

6. Nakahara W and Fukuoka F. Summation of carcinogenic effects of chemically unrelated carcinogens, 4-nitroquinoline $\mathrm{N}$-oxide and 20-methylcholanthrene. Gann. 51: 125-137. 1960.

7. Montesano R, Saffiotti U, Ferrero A, and Kaufman DG. Synergistic effects of benzo(alpha)pyrene and diethylnitrosamine on respiratory carcinogenesis in hamsters. J Natl Cancer Inst. 53: 1395-1397. 1974.

8. Habs $M$ and Schmahl D. Synergistic effects of N-nitroso compounds in experimental long-term carcinogenesis studies. Oncology. 37: 259-265. 1980.

9. Tsuda H, Miyata Y, Murasaki G, Kinoshita H, and Fukushima S. Synergistic effect of urinary bladder carcinogenesis in rats treated with N-butyl-n-(4hydroxybutyl)nitrosamine, N-(4-(5-nitro-2-furyl)-2thiazolyl)formamide, N-2-fluorenylacetamide, and 3,3'dichlorobenzidine. Gann. 68: 183-192. 1977.

10. Berger MR, Schmahl D, and Zerban H. Combination experiments with very low doses of three genotoxic Nnitrosamines with similar organotropic carcinogenicity in rats. Carcinogenesis. 8: 1635-1643. 1987.

11. Takayama S, Hasegawa H, and Ohgaki H. Combination effects of forty carcinogens administered at low doses to male rats. Jpn J Cancer Res. 80: 732-736. 1989.
12. Thomas GA and Williams ED. Thyroid stimulating hormone (TSH)-associated follicular hypertrophy and hyperplasia as a mechanism of thyroid carcinogenesis in mice and rats. IARC Sci Publ. 45-59. 1999.

13. Hasegawa R, Shirai T, Hakoi K, Wada S, Yamaguchi K, and Takayama S. Synergistic enhancement of thyroid tumor induction by 2,4-diaminoanisole sulfate, N, N'diethylthiourea and 4,4'-thiodianiline in male F344 rats. Carcinogenesis. 12: 1515-1518. 1991.

14. Ito $\mathrm{N}$, Tamano $\mathrm{S}$, and Shirai $\mathrm{T}$. A medium-term rat liver bioassay for rapid in vivo detection of carcinogenic potential of chemicals. Cancer Sci. 94: 3-8. 2003.

15. Shirai T, Hirose M, and Ito N. Medium-term bioassays in rats for rapid detection of the carcinogenic potential of chemicals. In: The Use of Short- and Medium-term Tests for Carcinogens and Data on Genetic Effects in Carcinogenic Hazard Evaluation. McGregor DB, Rice JM and Venitt S (eds). International Agency for Research on Cancer, Lyon. 251-272. 1999.

16. Ito $\mathrm{N}$, Hasegawa $\mathrm{R}$, Imaida $\mathrm{K}$, Hirose $\mathrm{M}$, and Shirai $\mathrm{T}$. Medium-term liver and multi-organ carcinogenesis bioassays for carcinogens and chemopreventive agents. Exp Toxic Pathol. 48: 113-119. 1996.

17. Ito N, Imaida $\mathrm{K}$, Asamoto $\mathrm{M}$, and Shirai T. Early detection of carcinogenic substances and modifiers in rats. Mutat Res. 462: 209-217. 2000.

18. Hirose M, Takesada $\mathrm{Y}$, Tanaka $\mathrm{H}$, Tamano S, Kato T, and Shirai T. Carcinogenicity of antioxidants BHA, caffeic acid, sesamol, 4-methoxyphenol and catechol at low doses, either alone or in combination, and modulation of their effects in a rat medium-term multi-organ carcinogenesis model. Carcinogenesis. 19: 207-212. 1998.

19. Doi K, Wanibuchi H, Salim EI, Shen J, Wei M, Mitsuhashi M, Kudoh S, Hirata K, and Fukushima S. Revised rat multiorgan carcinogenesis bioassay for whole-body detection of chemopreventive agents: modifying potential of Smethylcysteine. Cancer Lett. 206: 15-26. 2004.

20. Ito N, Hasegawa R, Imaida K, Kurata Y, Hagiwara A, and Shirai T. Effect of ingestion of 20 pesticides in combination at acceptable daily intake levels on rat liver carcinogenesis. Food Chem Toxicol. 33: 159-163. 1995.

21. Ito N, Hagiwara A, Tamano S, Hasegawa R, Imaida K, Hirose M, and Shirai T. Lack of carcinogenicity of pesticide mixtures administered in the diet at acceptable daily intake (ADI) dose levels in rats. Toxicol Lett. 82-83: 513-520. 1995.

22. Sugimura T, Wakabayashi K, Nakagama H, and Nagao M. Heterocyclic amines: Mutagens/carcinogens produced during cooking of meat and fish. Cancer Sci. 95: 290-299. 2004.

23. Ito N, Hasegawa R, Shirai T, Fukushima S, Hakoi K, Takaba K, Iwasaki S, Wakabayashi K, Nagao M, and Sugimura T. Enhancement of GST-P positive liver cell foci development by combined treatment of rats with five heterocyclic amines at low doses. Carcinogenesis. 12: 767-772. 1991.

24. Hasegawa R, Shirai T, Hakoi K, Takaba K, Iwasaki S, Hoshiya T, Ito N, Nagao M, and Sugimura T. Synergistic enhancement of glutathione S-transferase placental formpositive hepatic foci development in diethylnitrosaminetreated rats by combined administration of five heterocyclic amines at low doses. Jpn J Cancer Res. 82: 1378-1384. 1991. 
25. Hasegawa R, Kato T, Hirose M, Takahashi S, Shirai T, and Ito N. Enhancement of hepatocarcinogenesis by combined administration of food-derived heterocyclic amines at low doses in the rat. Food Chem Toxicol. 34: 1097-1101. 1996.

26. Fukushima S, Shibata MA, Hirose M, Kato T, Tatematsu M, and Ito N. Organ-specific modification of tumor development by low-dose combinations of agents in a rat wide-spectrum carcinogenesis model. Jpn J Cancer Res. 82: 784-792. 1991.

27. Hutt JA, Vuillemenot BR, Barr EB, Grimes MJ, Hahn FF, Hobbs CH, March TH, Gigliotti AP, Seilkop SK, Finch GL, Mauderly JL, and Belinsky SA. Life-span inhalation exposure to mainstream cigarette smoke induces lung cancer in $\mathrm{B} 6 \mathrm{C} 3 \mathrm{~F} 1$ mice through genetic and epigenetic pathways. Carcinogenesis. 26: 1999-2009. 2005.

28. Hecht SS. Carcinogenicity studies of inhaled cigarette smoke in laboratory animals: old and new. Carcinogenesis. 26: 1488-1492. 2005.

29. Fukushima S, Kinoshita A, Puatanachokchai R, Kushida M, Wanibuchi H, and Morimura K. Hormesis and doseresponse-mediated mechanisms in carcinogenesis: evidence for a threshold in carcinogenicity of non-genotoxic carcinogens. Carcinogenesis. 26: 1835-1845. 2005.

30. Ashby J, Elliott BM, Lefevre PA, Styles J, and Longstaff E. Initiation/promotion versus complete carcinogenicity in the rodent liver. Environ Health Perspect. 50: 339-346. 1983. 